\title{
Differential patterns of performance on the DKEFS in the presence of pediatric mood disturbances
}

\author{
Rachel K. Peterson ${ }^{1}$ - Chad A. Noggle ${ }^{2}$ - Jon C. Thompson ${ }^{3}$ \\ Mark T. Barisa ${ }^{4}$ - Alyssa Maulucci ${ }^{5}$ \\ ${ }^{1}$ Department of Counseling and Educational Psychology, Indiana University, Bloomington \\ ${ }^{2}$ Department of Psychiatry, Southern Illinois University School of Medicine, Springfield \\ ${ }^{3}$ Neuropsychology Department, St. Vincent Hospital, Indianapolis \\ ${ }^{4}$ Neuropsychology Department, Baylor Institute for Rehabilitation, Dallas \\ ${ }^{5}$ Department of Mental and Behavioral Health, VA Medical Center, Washington
}

DOI: http://dx.doi.org/10.7358/neur-2017-022-pete

rkpeters@imail.iu.edu

\section{ABSTRACT}

The current study investigated the differential patterns of performance on the DelisKaplan Executive Function System (D-KEFS) in pediatric mood disturbance in order to not only identify these profiles but also to suggest a utility of the D-KEFS in differentiating such presentations. An archival data set was utilized. Data were collected as part of a standard clinical practice at a Midwestern hospital. Participants included 19 children diagnosed with mood disturbances who completed the D-KEFS as part of a more comprehensive neuropsychological evaluation. Ratings were compared against normative sample means. Statistical analyses revealed mood disturbances in children was in fact related to a diminished function across a number of tasks on the D-KEFS as a sum, suggesting a broader compromise of executive functioning. Specific significant deficits $(p<.05)$ were noted on a number of measures. Purpose and use of evaluation of the scope of executive dysfunction in pediatric mood disturbances are discussed.

Keywords: Mood; Pediatrics; Executive function

Neuropsychological Trends - 22/2017

http://www.ledonline.it/neuropsychologicaltrends/ 


\section{INTRODUCTION}

Childhood mood disorders such as depression, anxiety, and bipolar disorder have been found to among children and adolescents and are not exclusive to the adult population. Per the American Psychiatric Association (2000), a mood disorder results in significant distress or impairment on a child's life and functioning. Best estimates for point prevalence rates for depressive disorders are $2.8 \%$ for children younger than 13 years old and $5.6 \%$ for 14 - to 18 -yearolds (Costello, Erkanli, \& Angold, 2006), with those aged 8 and younger showing rates less than 1\% (Keenan, Hipwell, Duax, Stouthamer-Loeber, \& Loeber, 2004). In terms of lifetime prevalence, rates up to $25 \%$ before adulthood have been found (Kessler, Avenevoli, \& Merikangas, 2001).

Rates of mania and bipolar disorder in youth are much lower than those in depressive disorders, and the disorder itself appears to be rare in children (Weckerly, 2002). A study by Lewinsohn, Klein, and Seeley (1995) found an estimated lifetime prevalence of $1 \%$ for bipolar disorders, with almost $6 \%$ of the sample reporting subclinical levels of bipolar symptoms (Lewinsohn, Zinbarg, Seeley, Lewinsohn, \& Sack, 1997). Other adolescent samples have yielded similar rates (Verhulst, van der Ende, Ferdinand, \& Kasius, 1997), but much lower rates have been found in preadolescents at approximately 0.1\% (Costello et al., 1996; Kessler \& Walters, 1998). The most common type of co-occurring disorder in youth with mood disorders are anxiety disorders. The 12-month prevalence ranges from $1-11 \%$, depending on the specific type of anxiety (DSM-V).

Cognition refers to the processes related to thinking and includes the ability to pay attention, retain and recall information, process information, solve problems, organize information, communicate, and act upon information (Miller \& Wallis, 2009). Deficits in cognitive functioning include difficulty with working memory, executive functioning, attention, and information processing, all of which have academic, social, and emotional implications. For example, impairments in memory and problem solving are associated with greater problems with independent living (Jefferson, Paul, Ozonoff, \& Cohen, 2006; Cahn-Weiner et al., 2007). Problems with attention, concentration, and thinking can make it difficult to keep up with schoolwork (Biederman et al., 2004; Breslau, Michael, Nancy, \& Ronald, 2008). Research has also shown that people with deficits across the domains of memory, problem solving, processing speed, and attention are more likely to be unemployed or have a lower occupational status (Baune et al., 2010; Hackman, Gallop, Evans, \& Farah, 2015). Furthermore, the ability to pay attention and focused is important for overall social functioning (Hungerford, Gonyo, Whitford, \& Bassendowski, 2009).

Limited research to date has examined how mood disturbances may impact cognitive functioning. A study by Pavuluri, West, Hill, Jindal, and Sweeney (2009) found weaknesses in executive functioning, verbal memory, attention, working 
memory visual memory, and visuospatial perception tasks relative to controls, all of which have potential lifelong implications for reduced cognitive functioning in adulthood. Favre and colleagues (2008) found similar results in children and adolescents diagnosed with major depressive disorder (MDD). Specifically, processing speed was decreased in the MDD versus control group and 27\% of the depressed group performed below average on the Trail Making Test.

Current neuroimaging studies point to a correlation between mood disorders and abnormalities in the left prefrontal and frontal cortices, portions of the temporal lobes, the amygdala, and the cingulate gyrus (Drevets, 2000, 2001; Vostrikov, Uranova, \& Orlovskaya, 2007). Given that neuroimaging findings of prefrontal and frontal cortex abnormalities, the area of executive functioning stands out as a likely neurocognitive manifestation of these abnormalities. As such, the purpose of the current study was to investigate the nature and extent of executive dysfunction, as manifested on the D-KEFS, in a sample of children with mood disturbances.

\section{METHOD}

The Delis-Kaplan Executive Function System (D-KEFS; Delis, Kaplan, \& Kramer, 2001) is an administered assessment of individual higher-level cognitive functioning skills, including flexibility of thinking, inhibition, problem solving, impulse control, concept formation, abstract thinking, and fluency. These tests were co-normed on a large and representative national sample designed exclusively for the assessment of executive functions including flexibility of thinking, inhibition, problem solving, planning, impulse control, concept formation, abstract thinking, and verbal creativity. The D-KEFS, composed of nine stand-alone tests that can be individually or group administered, provides a standardized assessment of executive functions in children and adults between the ages of 8 and 89 . The D-KEFS was developed to provide (a) measures that assess both fundamental and higher-level skills in order to evaluate if a patient's poor performance on a task is due to an executive-function deficit or to an impairment in a more fundamental cognitive skill, (b) strategies and error types in order to more precisely characterize the nature of the executive dysfunction, and (c) a form of test-design features that enhanced the instruments' sensitivity to mild executive dysfunction or mild frontal-lobe injury.

Each of the nine subtests assesses as aspect of verbal or non-verbal executive functioning (Fine \& Delis, 2011). The D-KEFS Trail-Making Test consists of five conditions: visual scanning, motor speed, number sequencing, letter sequencing, and number-letter switching. The four base-line conditions enable the clinician to assess empirically if a patient's poor performance on the switching condition is due to a deficit in cognitive flexibility or impairment in 
one or more of the underlying component skills needed to perform the switching task (i.e., motor speed, visual scanning, number sequencing, or letter sequencing). The D-KEFS Verbal Fluency Test is a timed measure that includes (a) a letter fluency condition that requires examinees to generate as many words as possible that start with a particular letter, (b) a category fluency condition that requires examinees to generate as many words as possible from designated semantic categories, and (c) a category switching condition that requires examinees to alternate between generating words from two different semantic categories. The D-KEFS Design Fluency Test was developed as a nonverbal analog of verbal fluency measures. The D-KEFS Sorting Test was designed to provide a standardized measure of conceptual-reasoning skills. Performance is evaluated both in terms of the total number of correct target concepts reflected in the examinee's sorts, as well as the accuracy and level of abstraction of the examinee's sort descriptions. The D-KEFS Twenty Questions Test assesses categorical processing, hypothesis testing, and concept formation. Multiple process measures are scored, including level of abstract thinking, error types, and response strategies (e.g., verbal versus nonverbal). The D-KEFS Word Context Test is a measure of deductive reasoning and verbal abstract thinking. In the D-KEFS Towers Test, the examinee is asked to build target towers by moving two to five disks of different sizes across three pegs in as few moves as possible while adhering to two rules: (a) move only one disk at a time and (b) never placing a larger disk over a smaller disk. The DKEFS Tower Test assesses several aspects of executive functioning, including spatial planning, rule violations, and inhibition. The D-KEFS Proverb Test measures metaphorical thinking and provides a means for comparing generation versus comprehension of abstract verbal information.

The standardized assessment of the D-KEFS was administered as part of clinical examination in a pediatric neuropsychology department as part of a Midwestern hospital. Institutional Review Board (IRB) approval was obtained for secondary analysis of data. For the purpose of this study, the researchers used a retrospective group-comparison design. That is, all diagnoses of mood disorders were made in conjunction with the cognitive assessment (D-KEFS) by use of other/independent measures as part of a larger, comprehensive neuropsychological assessment. The participants included 62 children and adolescents; $42(67.7 \%)$ male and $20(32.3 \%)$ female. During the course of this study, subjects were between the ages of eight and 19 years old (mean $=14.76$, $\mathrm{SD}=2.832)$, with an education between three and 13 years $($ mean $=8.89$, SD $=2.733)$. Fifty-five $(88.7 \%)$ of the subjects were right-handed, six $(9.7 \%)$ were left-handed, and one individual $(1.6 \%)$ was ambidextrous. On a self-report measure of ethnicity, $52(83.9 \%)$ individuals labeled themselves as Caucasian, 4 (6.5\%) were Hispanic, one (1.6\%) was African American, three (4.8) listed themselves as 'other', and two (3\%) chose to not identify themselves. 


\section{RESULTS}

Compared to normative rates, statistical analyses revealed mood disturbances in children were related to a diminished function across a number of tasks on the DKEFS as a sum, suggesting a broader compromise of executive functioning (Table 1). Specific significant deficits $(\mathrm{p}<.05)$ were noted on number sequencing, letter sequencing, letter-number sequencing, and motor speed of the Trails test; the inhibition condition of the color-word task; Condition 1 of design fluency; and sorting confirmed correct score. In addition, a multitude of significant findings were noted on the various supplemental calculations offered (Table 2).

Table 1. DKEFS outcomes in pediatric mood disturbance

\begin{tabular}{lcc}
\hline \multicolumn{1}{c}{ DKEFS Outcome } & $\begin{array}{c}\text { Mean } \\
\text { Difference }\end{array}$ & Sig. \\
\hline Trail Making: Visual Scanning & -1.206 & 0.104 \\
Trail Making: Number Sequencing & -2.500 & 0.005 \\
Trail Making: Letter Sequencing & -1.625 & 0.043 \\
Trail Making: Number-Letter Sequencing & -3.567 & $<0.001$ \\
Trail Making: Motor Speed & -0.750 & 0.249 \\
Color-Word: Color Naming & -1.167 & 0.029 \\
Color-Word: Word Reading & -0.500 & 0.288 \\
Color-Word: Inhibition & -1.559 & 0.019 \\
Color-Word: Inhibition/Switching & -1.000 & 0.107 \\
Verbal Fluency: Condition 1 & -1.929 & 0.060 \\
Verbal Fluency: Condition 2 & -1.357 & 0.058 \\
Verbal Fluency: Condition 3 & -0.357 & 0.307 \\
Verbal Fluency: Total Correct & 0.929 & 0.051 \\
Design Fluency: Condition 1 & -1.786 & 0.010 \\
Design Fluency: Condition 2 & -1.500 & 0.117 \\
Design Fluency: Condition 3 & 0.643 & 0.317 \\
Design Fluency: Total Correct & -1.071 & 0.167 \\
Sorting: Confirmed Correct & 2.900 & 0.037 \\
Sorting: Free Sorting & 4.000 & 0.022 \\
Sorting: Sorting Recognition Description & 0.500 & 0.426 \\
Sorting: Combined Description Score & 5.167 & 0.014 \\
Tower: Total Achievement & 0.100 & 0.452 \\
Tower: Mean First Move Time & 0.722 & 0.297 \\
\hline
\end{tabular}


Table 2. Additional significant supplemental calculations

\begin{tabular}{lcc}
\hline \multicolumn{1}{c}{ DKEFS Outcomes } & Mean Difference & Sig. \\
\hline $\begin{array}{l}\text { Trail Making: Switching vs. Visual Scanning } \\
\text { (Contrast Score) }\end{array}$ & -2.286 & 0.031 \\
$\begin{array}{l}\text { Trail Making: Switching vs. Combined } \\
\text { (Contrast Score) }\end{array}$ & -1.643 & 0.038 \\
$\begin{array}{l}\text { Trail Making: Switching vs. Motor Speed } \\
\text { (Contrast Score) }\end{array}$ & -3.143 & 0.006 \\
$\begin{array}{l}\text { Verbal Fluency: Category Switching vs. fluency } \\
\text { (Contrast Score) }\end{array}$ & 1.550 & 0.002 \\
$\begin{array}{l}\text { Verbal Fluency: Second Interval } \\
\text { Verbal Fluency: Category Switching (Percent }\end{array}$ & -1.833 & 0.016 \\
$\begin{array}{l}\text { Switching Accuracy) } \\
\begin{array}{l}\text { Design Fluency: Switching vs. Combined } \\
\text { (Contrast Score) }\end{array}\end{array}$ & 2.000 & 0.017 \\
$\begin{array}{l}\text { Design Fluency: Total Set Loss } \\
\text { Design Fluency: Total Repeated }\end{array}$ & 2.000 & 0.045 \\
\hline
\end{tabular}

\section{DISCUSSION}

The majority of research to date has demonstrated a reliance on executive functions on various other outcomes including academic achievement (St. Clair-Thompson \& Gathercole, 2006) and adaptive functioning (Clark, Prior, \& Kinsella, 2002). Few studies, however, have examined the role of mood on executive functioning. The current findings expand research along this line demonstrating that mood disturbances in children are associated with an array of difficulties in executive control, including difficulties with sequencing, mental flexibility, fluency, and problem solving. The establishment of deficits on the D-KEFS within this clinical group may suggest its utility in differentiating mood disturbances from other presentations in pediatric populations, barring additional investigations.

Further, current findings illustrate the importance of intervention along executive lines for children with depression and the utility and efficacy of neuropsychological assessment to guide interventions and treatment planning.

For example, neuropsychologists may want to examine executive functioning in individuals who present for clinical assessment with a mood 
disorder. Treatment that includes not only cognitive-behavioral therapy (CBT) and pharmacological intervention but also improves upon executive functioning weaknesses appears warranted as well.

\section{REFERENCES}

American Psychiatric Association. (2000). Diagnostic and statistical manual of mental disorders, fourth edition (text revision). Washington, DC: American Psychiatric Association.

Baune, B. T., Miller, R., McAfoose, J., Johnson, M., Quirk, F., \& Mitchell, D. (2010). The role of cognitive impairment in general functioning in major depression. Psychiatry Research, 176(2), 183-189.

Biederman, J., Monuteaux, M. C., Doyle, A. E., Seidman, L. J., Wilens, T. E., Ferrero, F., ... \& Faraone, S. V. (2004). Impact of executive function deficits and attention-deficit/hyperactivity disorder (ADHD) on academic outcomes in children. Journal of Consulting and Clinical Psychology, 72(5), 757.

Breslau, J., Lane, M., Sampson, N., \& Kessler, R. C. (2008). Mental disorders and subsequent educational attainment in a US national sample. Journal of Psychiatric Research, 42(9), 708-716.

Cahn-Weiner, D. A., Farias, S. T., Julian, L., Harvey, D. J., Kramer, J. H., Reed, B. R., ... \& Chui, H. (2007). Cognitive and neuroimaging predictors of instrumental activities of daily living. Journal of the International Neuropsychological Society, 13(05), 747-757.

Clark, C., Prior, M., \& Kinsella, G. (2002).The relationship between executive function abilities, adaptive behaviour, and academic achievement in children with externalizing behaviour problems. Journal of Child Psychology and Psychiatry, 43(6), 785-796

Costello, E. J., Angold, A., Burns, B. J., Stangl, D. K., Tweed, D. L., Erkanli, A., \& Worthman, C. M. (1996). The Great Smoky Mountains study of youth: Goals, design, methods, and prevalence of DSM-III-R disorders. Archives of General Psychiatry, 53(12), 1129-1136.

Costello, E. J., Erkanli, A., \& Angold, A. (2006). Is there an epidemic of child or adolescent depression? Journal of Child Psychology and Psychiatry, 47(12), 1263-1271.

Delis, D. C., Kaplan, E., \& Kramer, J. H. (2001). Delis-Kaplan executive function system (D-KEFS). Psychological Corporation.

Drevets, W. C. (2000). Neuroimaging studies of mood disorders. Biological Psychiatry, 48(8), 813-829. 
Drevets, W. C. (2001). Neuroimaging and neuropathological studies of depression: implications for the cognitive-emotional features of mood disorders. Current Opinion in Neurobiology, 11(2), 240-249.

Favre, T., Hughes, C., Emslie, G., Stavinoha, P., Kennard, B., \& Carmody, T. (2008). Executive functioning in children and adolescents with major depressive disorder. Child Neuropsychology, 15(1), 85-98.

Fine, E. M., \& Delis, D. C. (2011). Delis-Kaplan Executive Functioning System. In Encyclopedia of Clinical Neuropsychology, (pp. 796-801). New York, NY: Springer.

Keenan, K., Hipwell, A., Duax, J., Stouthamer-Loeber, M., \& Loeber, R. (2004). Phenomenology of depression in young girls. Journal of the American Academy of Child \& Adolescent Psychiatry, 43(9), 1098-1106.

Kessler, R. C., \& Walters, E. E. (1998). Epidemiology of DSM-III-R major depression and minor depression among adolescents and young adults in the National Comorbidity Survey. Depression and Anxiety, 7(1), 3-14.

Kessler, R. C., Avenevoli, S., \& Merikangas, K. R. (2001). Mood disorders in children and adolescents: An epidemiologic perspective. Biological Psychiatry, 49(12), 1002-1014.

Lewinsohn, P. M., Klein, D. N., \& Seeley, J. R. (1995). Bipolar disorders in a community sample of older adolescents: Prevalence, phenomenology, comorbidity, and course. Journal of the American Academy of Child and Adolescent Psychiatry, 34(4), 454-463.

Lewinsohn, P. M., Zinbarg, R., Seeley, J. R., Lewinsohn, M., \& Sack, W. H. (1997). Lifetime comorbidity among anxiety disorders and between anxiety disorders and other mental disorders in adolescents. Journal of Anxiety Disorders, 11(4), 377-394.

Hackman, D. A., Gallop, R., Evans, G. W., \& Farah, M. J. (2015). Socioeconomic status and executive function: developmental trajectories and mediation. Developmental Science, 18(5), 686-702.

Hungerford, S., Gonyo, K., Whitford, S., \& Bassendowski, N. (2009). Executive Functions and Their Relationship to Social Skills and Problem Behaviors. Poster session presented at the annual meeting of the New York Speech Language Hearing Association, Saratoga Springs, NY.

Jefferson, A. L., Paul, R. H., Ozonoff, A., \& Cohen, R. A. (2006). Evaluating elements of executive functioning as predictors of instrumental activities of daily living (IADLs). Archives of Clinical Neuropsychology, 21(4), 311-320.

Miller, E. K., \& Wallis, J. D. (2009). Executive function and higher-order cognition: definition and neural substrates. Encyclopedia of Neuroscience, 4, 99-104. 
Pavuluri, M. N., West, A., Hill, S. K., Jindal, K., \& Sweeney, J. A. (2009). Neurocognitive function in pediatric bipolar disorder: 3-year follow-up shows cognitive development lagging behind healthy youths. Journal of the American Academy of Child \& Adolescent Psychiatry, 48(3), 299-307.

St Clair-Thompson, H. L, \& Gathercole, S. E. (2006). Executive functions and achievements in school: Shifting, updating, inhibition, and working memory. The Quarterly Journal of Experimental Psychology, 59(4), 745-759.

Verhulst, F. C., van der Ende, J., Ferdinand, R. F., \& Kasius, M. C. (1997). The prevalence of DSM-III-R diagnoses in a national sample of Dutch adolescents. Archives of General Psychiatry, 54(4), 329-336.

Vostrikov, V. M., Uranova, N. A., \& Orlovskaya, D. D. (2007). Deficit of perineuronal oligodendrocytes in the prefrontal cortex in schizophrenia and mood disorders. Schizophrenia Research, 94(1), 273-280.

Weckerly, J. (2002). Pediatric bipolar mood disorder. Journal of Developmental \& Behavioral Pediatrics, 23(1), 42-56. 
